\title{
NK/T cell non-Hodgkin lymphoma in a HIV-positive patient
}

\author{
Nicole C. Panarelli • Richard R. Furman • \\ Y. Lynn Wang • Rebecca Elstrom • Jules A. Cohen • \\ Amy Chadburn
}

Received: 9 November 2009 / Accepted: 8 February 2010/Published online: 4 March 2010

(C) Springer-Verlag 2010

\begin{abstract}
NK/T lymphomas have rarely been reported in HIV/AIDS patients. Here we report a case of a 37-year-old woman, with AIDS and a recent diagnosis of Kaposi sarcoma in a mesenteric lymph node, who presented with extra-ocular nerve palsies and gastrointestinal bleeding. A small intestine resection specimen revealed an extra-nodal NK/T cell lymphoma, nasal type. The unique presentation of this rare and aggressive lymphoma in the setting of AIDS and Kaposi sarcoma underscores the importance of maintaining a broad differential diagnosis when evaluating a malignant neoplasm from a HIV-positive patient.
\end{abstract}

Keywords Natural killer/T cell lymphoma · AIDS · HIV · Kaposi sarcoma

\section{Introduction}

Non-Hodgkin lymphoma is the second most common neoplasm occurring in patients with HIV/AIDS. The majority are aggressive B cell lymphomas that also occur

N. C. Panarelli $\cdot$ Y. L. Wang

Department of Pathology and Laboratory Medicine, Weill Cornell Medical College,

New York, NY, USA

R. R. Furman · R. Elstrom · J. A. Cohen

Department of Hematology and Medical Oncology,

Weill Cornell Medical College,

New York, NY, USA

\section{A. Chadburn $(\bowtie)$}

Department of Pathology,

Northwestern University Feinberg School of Medicine,

Chicago, IL, USA

e-mail: achadburn@northwestern.edu in immunocompetent patients, i.e., Burkitt lymphoma and diffuse large B cell lymphoma [1]. Additional B cell lymphomas occur predominately in HIV/AIDS patients, but only rarely in immunocompetent patients, and include primary effusion lymphoma (PEL), plasmablastic lymphoma of the oral cavity, and Kaposi sarcoma herpesvirus (KSHV; HHV-8) associated lymphomas arising in the setting of multicentric Castleman disease $[1,2]$. In contrast to immunocompetent patients, many of the lymphomas in HIV/AIDS patients are associated with herpes viruses, particularly Epstein-Barr virus (EBV) and KSHV. HIV/ AIDS patients with lymphoma often present with extranodal disease, most frequently involving the gastrointestinal tract, central nervous system, bone marrow, and liver [1].

Natural killer (NK)/T cell lymphoma, nasal type, is a relatively rare disease process seen most frequently in Asia, South and Central America, and only occasionally in Western countries. In most cases these neoplasms display an immunophenotype and genotype typical of NK-cell lymphomas (EBV positive, CD56 positive, CD2 positive, cytoplasmic $\mathrm{CD} 3$ positive, surface $\mathrm{CD} 3$ negative, cytotoxic granule positive; no $\mathrm{T}$ cell receptor gene rearrangement). However, some lesions that may be EBV positive, cytotoxic granule positive, but CD56 negative and surface CD3 positive are also considered NK/T lesions [3, 4]. In addition, a few cases exhibit a monoclonal $\mathrm{T}$ cell receptor gene rearrangement; these cases are thought to correspond to NK/T cell lymphomas of cytotoxic $\mathrm{T}$ cell origin [3]. Thus, while there are NK/T cell lymphomas that are of true NK origin, there are others that are phenotypically and genotypically similar to cytotoxic T cells $[3,4]$. Morphologically these aggressive tumors are characterized by angiocentric lymphoid infiltrates, vascular destruction, and necrosis. NK/T cell lymphomas present most commonly in the nasal cavity, but can also occur in other, particularly 
extra-nodal, sites including the skin, gastrointestinal tract, orbit, and testes [3].

Occasionally NK/T cell lymphomas occur in immunodeficient or immunosuppressed patients including transplant recipients, however, they have only rarely been diagnosed HIV/AIDS patients. The NK/T cell lymphoma cases in HIV/AIDS have varied extensively with respect to immunophenotype, sites of disease, and clinical presentation [5-11]. We report here a unique case of $\mathrm{NK} / \mathrm{T}$ cell lymphoma arising in the gastrointestinal tract and central nervous system of a severely immunodeficient HIV-positive woman with biopsy proven Kaposi sarcoma (KS). Although in the clinical setting of KS, HIV positivity and clinical AIDS, disseminated KS or an aggressive B cell lymphoma, including a KSHV/HHV-8-positive extracavitary PEL, would be more common, her rapid disease progression and the anatomic sites of involvement underscore the importance of including other processes, such as $\mathrm{NK} / \mathrm{T}$ cell lymphoma in the differential diagnosis.

\section{Clinical history}

The patient is a 37-year-old Hispanic woman who was born the United States with long-standing HIV/AIDS, biopsy confirmed low-grade squamous intraepithelial lesion of the cervix (last follow-up within a month of admission) and no history of opportunistic infections in spite being noncompliant with her anti-retroviral therapy. Her most recent CD4 count was 18 cells per milliliter with a viral load of 113,914 copies per milliliter. She presented to the emergency department in October 2007 complaining of persistent and increasing left-sided ptosis and diplopia. Admission laboratories showed a white blood cell count of $5.1 \mathrm{~K} / \mathrm{uL}$, hemoglobin of $11 \mathrm{~g} / \mathrm{dL}$, and a platelet count of $364 \mathrm{~K} / \mathrm{uL}$; no "blasts" or abnormal circulating cells were noted. On physical examination, no skin lesions, organomegaly or ascites were identified. An MRI on admission showed enhancement of the left third cranial nerve and subcortical T2 hyperintensities. A lumbar puncture showed EBV in the cerebrospinal fluid (CSF) by PCR analysis (205,000 DNA copies per milliliter); all other PCR studies for viruses in the CSF, including KSHV/HHV8, cytomegalovirus, herpes simplex virus, enterovirus, JC virus, and varicella zoster virus, were negative. Cytologic examination of the CSF showed no malignant cells; flow cytometry was not performed. The serum LDH was elevated to $353 \mathrm{U} / \mathrm{L}$ $(\mathrm{nl}<200 \mathrm{U} / \mathrm{L})$. A full body PET-CT scan on the third day after admission revealed hypermetabolic nonobstructive concentric wall thickening of a loop of small bowel with adjacent mesenteric fat stranding, SUV of 23; mesenteric lymphadenopathy up to $2.0 \times 1.3 \mathrm{~cm}$ in size with maximum SUV of 13.3; hypermetabolic activity in the left paraspinous muscles with corresponding disruption of the fat planes but no obvious mass lesion on CT, maximum SUV of 23.7; and left external iliac lymphadenopathy $1.9 \times 0.9 \mathrm{~cm}$ with maximum SUV of 4.3. Additionally, evaluation of the brain by PET-CT scan demonstrated abnormal focal hypermetabolic uptake localizing to the base of the skull, immediately posterior to the sphenoid sinuses, with a maximum SUV of 16.3. An ultrasound guided biopsy of a mesenteric lymph node showed Kaposi sarcoma confirmed by immunostaining for latent nuclear antigen (LANA; Advanced Biotechnologies, Inc., Columbia, MD, USA). On hospital day 4, the patient experienced an acute drop in hemoglobin (from 9.2 to $7.3 \mathrm{mg} / \mathrm{dL}$ ) and was noted to have a positive stool guaiac. Colonoscopy and upper endoscopy revealed no source of bleeding or other abnormality. Due to worsening ptosis and diplopia, a repeat MRI on hospital day 6 was performed which demonstrated progressive enhancement of the left third cranial nerve. Over the next 10 days, the patient experienced periodic decreases in hemoglobin level and blood pressure, requiring repeated transfusions of red blood cells and fluid resuscitation. Her cranial nerve palsy progressed to involve the right third cranial nerve as well as cranial nerves IV, V, and VI on the left. All blood cultures, including viral, were negative.

Given the recurrent episodes of bleeding and the need for more definitive pathologic diagnosis, the patient underwent an exploratory laparotomy with resection of the small bowel and removal of an abdominal wall implant. At the time of surgery multiple full-thickness ulcerations in association with small intestinal mural thickening were noted. Pathologic analysis of the small bowel and abdominal wall implant demonstrated NK/T cell lymphoma. Bone marrow biopsy performed following diagnosis was negative by morphology, flow cytometry, and immunohistochemical staining for lymphoma; peripheral blood analysis by flow cytometry was also negative for NK/T cell lymphoma but showed an inverted CD4/CD8 ratio of 0.1 . A repeat CSF evaluation was also negative for malignant cells. The patient was treated with high-dose methotrexate-based chemotherapy plus liposomal cytarabine through an ommaya port. A CT scan 12 months after presentation and after becoming compliant with her antiretroviral therapy showed no evidence of disease. However, approximately 16 months following her diagnosis of lymphoma the patient was found to have a high-grade squamous intraepithelial lesion.

\section{Methods}

Representative sections from ulcerated and thickened areas of the small bowel as well as the abdominal wall implant were routinely processed. Formalin-fixed, paraffinembedded tissue sections were stained with hematoxylin 
and eosin. In addition, sections of tumor were immunostained for BCL-2, BCL-6, kappa, lambda, latent membrane protein 1 (LMP-1), MUM-1, EBV nuclear antigen 2 (EBNA2), Ki67, epithelial membrane antigen (EMA), CD8, CD20, CD30 (Dako, Glastrup, Denmark), CD2, CD4, CD7, CD56 (Vision Biosystems Novocastra, New Castle-upon-Tyne, UK), CD15, CD57, PAX-5 (BectonDickinson, San Jose, CA, USA), CD5 (Vector Laboratories, Burlingame, CA, USA), LANA (Advanced Biotechnologies, Inc., Columbia, MD, USA), D2-40 (Convance, Berkeley, CA, USA), and CD3 (Thermo Fischer Scientific, Fremont, CA, USA). In situ hybridization for EBV was performed using an EBER probe (Leica Microsystems, Bannockburn, IL, USA) according to the manufacturer's instructions. All studies were performed on the Bond Max Immunostainer (Leica Microsystems, Bannockburn, IL, USA) using appropriate antigen retrieval or hybridization steps. PCR analysis was performed for the antigen receptor genes; the conditions for these PCR reactions have been previously described [2].

\section{Results}

Gross examination of the abdominal wall implant revealed firm fleshy nodules and a purulent exudate. Examination of the resected small bowel revealed several full-thickness ulcers measuring up to $2 \mathrm{~cm}$ in greatest dimension and surrounded on all sides by a green purulent exudate. The ulcers were associated with areas of nodular mural thickening. Cut sections of the thickened areas revealed white, fleshy, homogeneous surfaces (Fig. 1a).

Histologic sections of the abdominal wall mass and small intestine showed that the malignant cells focally
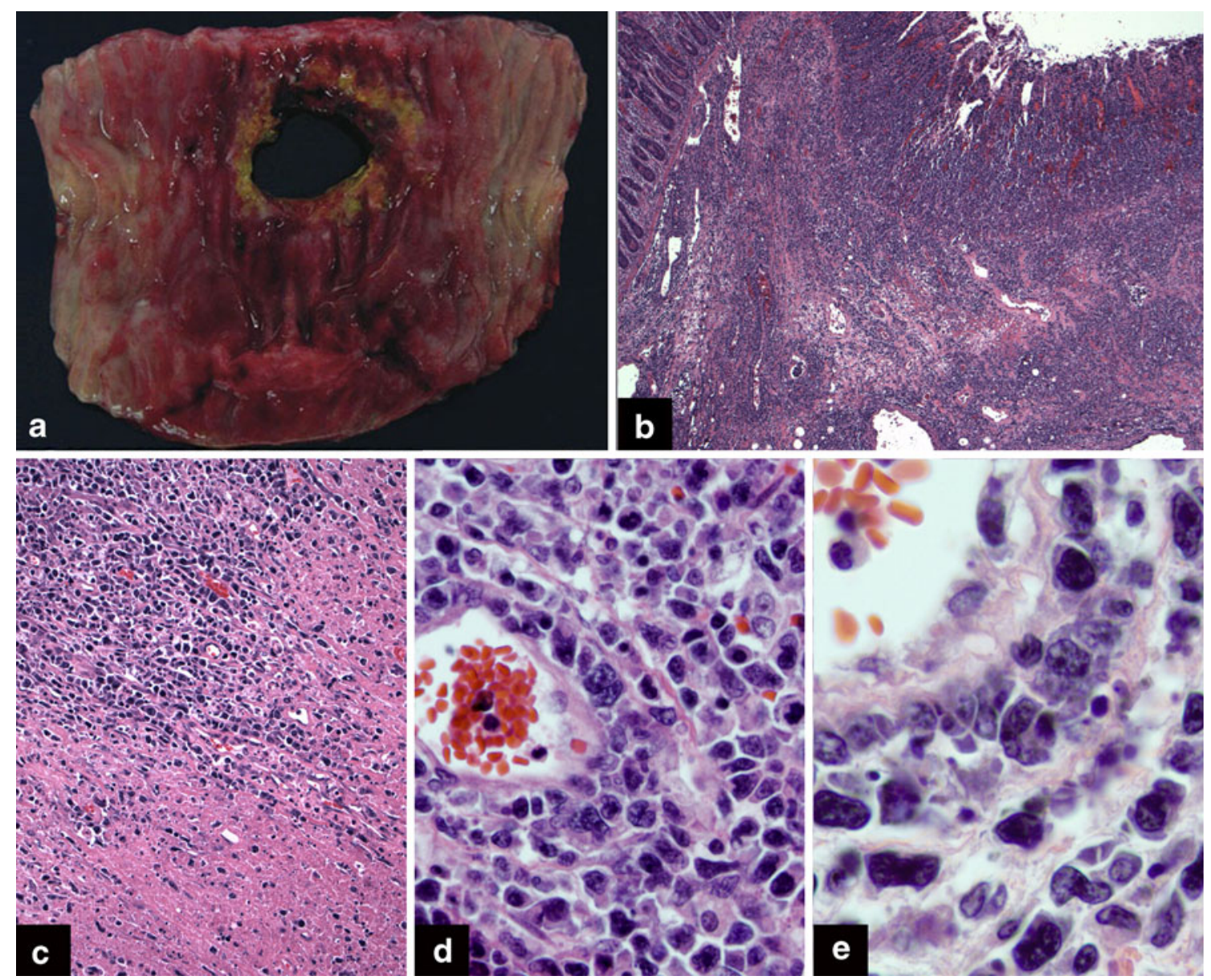

Fig. 1 a The segment of small intestine showed firm fleshy areas of mural thickening and several full-thickness ulcers surrounded by a purulent exudate. b There was an extensive, focally transmural infiltrate of large atypical lymphoid cells (hematoxylin and eosin $4 \times$ original magnification). c Areas of coagulative necrosis were present (hematoxylin and eosin $20 \times$ original magnification). d Focally the tumor cells were seen in the vessel walls. The cells had medium to large hyperchromatic nuclei with irregular nuclear contours and a scant to moderate amount of cytoplasm. Mitotic figures were easily identified (hematoxylin and eosin $50 \times$ original magnification). e At high power some of the cells were seen to be almost Reed-Sternberglike in appearance. The cells were pleomorphic with a variable number of prominent nucleoli. Apoptotic debris was seen (hematoxylin and eosin $100 \times$ original magnification) 
Fig. 2 Immunophenotypic analysis showed that the cells a were negative for the $\mathrm{B}$ cell-associated antigen, CD20, but $\mathbf{b}$ were $\mathrm{CD} 2$ and $\mathbf{c}$ CD56 positive. $\mathbf{d}$ The tumor cells were positive for the Epstein-Barr virus (EBV)-based on in situ hybridization using an EBER probe $(\mathbf{a}, \mathbf{b}, \mathbf{c}$ : immunoperoxidase $4 \times$ original magnification; $\mathbf{d}$ in situ hybridization $4 \times$ original magnification)

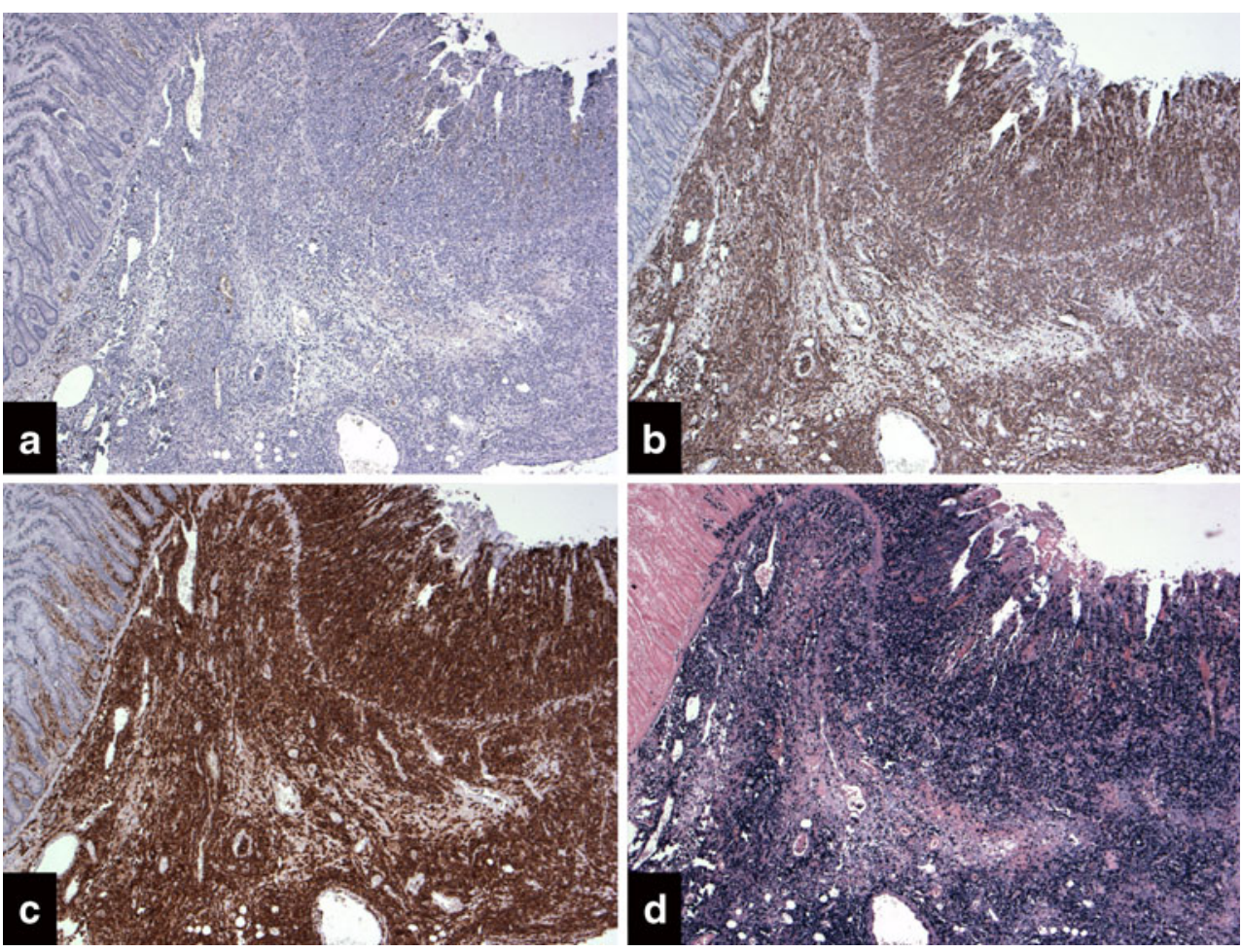

involved the full thickness of the bowel (Fig. 1b). Multiple mucosal ulcerations as well as areas of necrosis were also present (Fig. 1c). The infiltrate consisted of atypical pleomorphic medium (some) to large (most) lymphoid cells (Fig. 1d, e). The cells had irregular contours, one to multiple prominent nucleoli, and a scant to moderate amount of cytoplasm. A significant number of the cells were multi-lobated. The cells exhibited a high proliferation rate with numerous mitotic figures; apoptotic debris was abundant. Vascular invasion by the tumor cells was also present. Immunophenotypically, the tumor cells lacked expression of B cell-associated antigens such as CD20 (Fig. 2a) and PAX-5 and the follicular center cell-associated marker BCL-6. The cells were positive for the pan $\mathrm{T}$ cell markers CD2 (Fig. 2b), CD3 and CD7 but lacked expression of CD5. They were also negative for CD4 and CD8. The cells expressed the NK-cell-associated marker CD56 (Fig. 2c), but were negative for CD16 and CD57. The cells were also negative for CD15, but expressed CD30 and MUM-1. Immunostaining for the LANA of the KSHV/ HHV-8 was negative. In situ hybridization for EBV was positive (Fig. 2d). The cells also expressed the EBVassociated protein LMP-1, but lacked expression of EBNA2. This immunohistochemical profile is most consistent with that of an extra-nodal NK/T lymphoma, nasal type. PCR analysis showed no evidence of either an immunoglobulin heavy chain gene or $\mathrm{T}$ cell receptor gamma chain gene rearrangement.

\section{Discussion}

Natural killer/T cell lymphomas are uncommon lymphoid neoplasms that are usually diagnosed in persons from Asia and South America [3]. NK/T cell lymphomas have only rarely been diagnosed in patients with HIV/AIDS with fewer than ten cases reported in the literature [5-11]. Similar to our patient, two of the HIV/AIDS patients in the literature had CNS disease, including one who also had involvement of the orbit and the nasal/maxillary sinuses [7, 9]. The latter patient, a 42-year-old male, presented with double vision and eye-droop as did our patient. The primary sites of disease in the other HIV/AIDS patients with NK/T cell lymphoma reported in the literature include nasopharynx, tonsil, parotid gland, lung/mediastinum, and lymph node. However, in most of the patients, lymphoma was also identified in other sites, most commonly the liver, either at presentation or later in the course of their disease. Involvement of the colon, which occurs relatively frequently in HIV negative patients and was a major site of involvement in our patient, was not reported in the other seven cases identified in the literature [5-11].

All but one of the reported $\mathrm{NK} / \mathrm{T}$ cell lymphomas in HIV/AIDS patients have been diagnosed in men (median age of 42 years) with low CD4 counts $(<200 /$ dl, where reported) [5, 7-11]. The remaining case occurred in an 18month-old Chinese girl with perinatal HIV infection [6]. This child presented with an enlarged supraclavicular 
lymph node, which on biopsy revealed NK/T cell lymphoma. NK/T cell proliferations have been reported in children, termed severe chronic active EBV infection (CAEBV), but in general NK/T cell lymphomas only infrequently involve lymph nodes.

Interestingly, during the initial work-up of our patient, PET/CT scans showed mesenteric lymphadenopathy consistent with malignant lymphoma. A biopsy of a mesenteric lymph node, however, showed KS. The subsequent colon specimen, in contrast, contained a pleomorphic, high-grade large cell lymphoma. This finding, in a patient with KS, strongly suggested the possibility of a solid (extra-cavitary) PEL [2]. However, work-up showed that the lymphoma, although EBV positive, was not a KSHV/HHV-8 positive extra-cavitary PEL as the cells were negative for the $\mathrm{KSHV} / \mathrm{HHV}-8$-associated LANA and did not contain a clonal rearrangement of the immunoglobulin heavy chain gene, but instead expressed CD56, CD2, and CD3 and was EBV positive, consistent with an extra-nodal NK/T cell lymphoma, nasal type. Thus, this patient had two completely separate neoplastic processes, both of which are associated with different herpes virus infections.

NK/T cell lymphomas are aggressive neoplasms [3]. Of the previously reported HIV/AIDS patients with NK/T cell lymphoma reported in the literature, only one patient of the six with follow-up lived more than 1 month following diagnosis. The exception, a 42-year-old homosexual male, known to be HIV-positive for 14 years, who presented with parotid gland and liver disease and an absolute CD4 count of 0 and lived 22 months following diagnosis. He was treated with two rounds of chemotherapy, but did not complete the second course. He subsequently developed pulmonary nodules and died of probable lymphoma [8]. Our patient received combination chemotherapy with alleviation of symptoms and has survived more than 1 year. Her followup CT scan 12 months after presentation showed no evidence of disease. However, her clinical course may also be influenced by her improved immune status by remaining compliant with her HAART therapy.

Our understanding of NK/T cell lymphomas, particularly in the HIV/AIDS patient population, is limited particularly due to the relatively small number of cases diagnosed. Furthermore, the diagnosis of NK/T cell neoplasms is complicated by the significant phenotypic and genotypic overlap between NK cells and cytotoxic T cells and by lack of a "clonality" marker in NK cell proliferations. Recently described antigens on NK cells and some cytotoxic T cells termed "kill inhibitory receptors" (KIRs) are thought to protect potential targets from NK-cell-mediated lysis. KIRs are of particular interest because they are stably expressed over multiple generations and thus may serve as a marker for clonality. Studies have shown that in normal NK cell populations, varying portions of NK cells are positive for each of multiple KIR antigens whereas NK neoplasms show restricted KIR expression or no KIR expression at all. To date, studies that test the use of KIRs for this purpose have been small, owing to the scarcity of NK cell neoplasms, however these antigens show potential as diagnostic tools for these rare and aggressive neoplasms [12].

Our patient has experienced relatively long disease free survival. Not only is this unusual in patients with NK/T cell lymphoma, particularly one with HIV/AIDS, but is also surprising as our patient presented with other poor prognostic indicators including intestinal involvement, disseminated disease, high level of EBV DNA in the CSF, and a low CD4 count $[3,13,14]$. Although therapy for NK/T cell lymphoma has focused on anthracycline-based chemotherapy with or without radiation [13], because of the need to treat her CNS disease, a regimen utilizing methotrexate every 14 days was used [15]. Furthermore, as she presented with disseminated disease, radiation was thought to be most likely of little benefit. Thus, she did not receive radiation therapy despite the fact that at least some investigators think that it supplies the greatest part of treatment efficacy [16]. Although the impact of an improved CD4 count, secondary to HAART compliance, on survival is large, this patient's complete response and relatively long survival raise the possibility that NK/T cell lymphomas may be more sensitive to methotrexate-based treatment regimens than those employing anthracycline as well as implying that radiation therapy may not be as important as has been previously thought. However, randomized trials are needed to clarify these possibilities.

In this study, we describe a severely immunosuppressed HIV/AIDS woman with gastrointestinal and central nervous system extra-nodal NK/T cell lymphoma, nasal type, an unusual diagnosis in a patient population where the vast majority of the lymphoid neoplasms are aggressive B cell lymphomas such as diffuse large B cell lymphoma, Burkitt lymphoma and in patients with very low CD4 counts and known KS, PEL/extra-cavitary PEL [1, 2]. However, the anatomic sites of involvement and the very rapid disease progression underscore the importance of considering other disease processes, such as NK/T lymphoma, in the differential diagnosis.

Conflict of interest The authors declare that they have no conflict of interest.

\section{References}

1. Raphael M, Said J, Borisch B, Cesarman E, Harris NL (2008) Lymphomas associated with HIV infections. In: Swerdlow SH, Campo E, Harris NL, Jaffe E, Pileri SA, Stein H, Thiele J, Vardiman JW (eds) WHO Classification of Tumours of Haema- 
topoietic and Lymphoid Tissues. International Agency for Research on Cancer (IARC), Lyon (France), pp 340-342

2. Chadburn A, Hyjek E, Mathew S, Cesarman E, Said J, Knowles DM (2004) KSHV-positive solid lymphomas represent an extracavitary variant of primary effusion lymphoma. Am J Surg Pathol 28:1401-1416

3. Chan JKC, Quintanilla-Martinez L, Ferry JA, Peh S-C (2008) Extranodal NK/T-cell lymphoma, nasal type. In: Swerdlow SH, Campo E, Harris NL, Jaffe E, Pileri SA, Stein H, Thiele J, Vardiman JW (eds) WHO Classification of Tumours of Haematopoietic and Lymphoid Tissues. International Agency for Research on Cancer (IARC), Lyon (France), pp 285-288

4. Hasserjian RP, Harris NL (2007) NK-cell lymphomas and leukemias: a spectrum of tumors with variable manifestations and immunophenotype. Am J Clin Pathol 127:860-868

5. Canioni D, Arnulf B, Asso-Bonnet M, Raphael M, Brousse N (2001) Nasal natural killer lymphoma associated with EpsteinBarr virus in a patient infected with human immunodeficiency virus. Arch Pathol Lab Med 125:660-662

6. Chiu SS, Chan GC, Loong F (2001) Epstein-Barr virus (EBV) induced hemophagocytic syndrome followed by EBV associated T/NK lymphoma in a child with perinatal human immunodeficiency virus (HIV) infection. Med Pediatr Oncol 36:326-328

7. Cobo F, Talavera P, Busquier H, Concha A (2007) CNK/T-cell brain lymphoma associated with Epstein-Barr virus in a patient with AIDS. Neuropathology 27:396-402

8. Cornfield DB, Papiez JS, Lynch JT, Rimsza LM (2002) Natural killer-like T-cell lymphoma of the parotid in a patient infected with human immunodeficiency virus. Arch Pathol Lab Med 126:738-741

9. D'Souza GA, Sunad R, Rajagopalan N, Ananthamurthy A, Murthy KR, Babu K (2006) NK/T-cell lymphoma in AIDS. J Assoc Physicians India 54:890-892
10. Oh SC, Choi CW, Kim BS, Shin SW, Kim YH, Lee JS, Kim MJ, Jung WY, Kim HK, Yeom BW, Kim IS, Kim JS (2004) NK/T-cell lymphoma associated with Epstein-Barr virus in a patient infected with human immunodeficiency virus: an autopsy case. Int J Hematol 79:480-483

11. Tao J, Savargaonkar P, Vallejo C, Cesarman E, Fuchs A (2000) Aggressive natural killer cell lymphoma presenting as an anterior mediastinal mass in a patient with acquired immunodeficiency syndrome. Arch Pathol Lab Med 124:304-309

12. Morice WG (2007) The immunophenotypic attributes of NK cells and NK-cell lineage lymphoproliferative disorders. Am J Clin Pathol 127:881-886

13. Au WY, Weisenburger DD, Intragumtornchai $\mathrm{T}$, Nakamura $\mathrm{S}$, Kim WS, Sng I, Vose J, Armitage JO, Liang R (2009) Clinical differences between nasal and extranasal natural killer/T-cell lymphoma: a study of 136 cases from the International Peripheral T-Cell Lymphoma Project. Blood 113:3931-3937

14. Bower M, Gazzard B, Mandalia S, Newsom-Davis T, Thirlwell C, Dhillon T, Young AM, Powles T, Gaya A, Nelson M, Stebbing J (2005) A prognostic index for systemic AIDS-related nonHodgkin lymphoma treated in the era of highly active antiretroviral therapy. Ann Intern Med 143:265-273

15. Abrey LE, Yahalom J, DeAngelis LM (2000) Treatment for primary CNS lymphoma: the next step. J Clin Oncol 18:31443150

16. Lee J, Au WY, Park MJ, Suzumiya J, Nakamura S, Kameoka J, Sakai C, Oshimi K, Kwong YL, Liang R, Yiu H, Wong KH, Cheng HC, Ryoo BY, Suh C, Ko YH, Kim K, Lee JW, Kim WS, Suzuki R (2008) Autologous hematopoietic stem cell transplantation in extranodal natural killer/T cell lymphoma: A multinational, multicenter, matched controlled study. Biol Blood Marrow Transplant 14:1356-1364 